\title{
Research on Correction Method of Non - coherent Velocity Error
}

\author{
Mingxin Kou, Gang Fu and Wei Yu
}

China Satellite Maritime Tracking and Control Department, Jiangyin, 214431, China

Keywords: Frequency difference; noncritical; Speed.

\begin{abstract}
The measurement and control system works under the non-coherent system. When the nominal frequency of the star is not consistent, the velocity measurement results introduce the system error. In order to eliminate this kind of influence; this paper analyzes the influence of the inconsistency of the nominal frequency of the star from the basic principle and algorithm of the noncoherent velocity measurement. It is concluded that the nominal frequency difference measurement of the star is inevitable, Normalized to the station nominal downlink frequency, the frequency inconsistency part of the conversion to the star clock error, measured downstream nominal frequency normalized satellite nominal uplink frequency, the method for the speed system error correction, thereby improving the non-coherent speed Accuracy.
\end{abstract}

\section{Introduction}

XX satellite using non-coherent spread spectrum measurement and control system, YUANWANG 5 ship in the docking process found in the static conditions of the star speed test results are not zero. The details are as follows:

Satellite test equipment placed in the standard tower shielding cage, to participate in the satellite equipment to use radio frequency connection means to form a large loop, for speed performance test. Star equipment to work in the uplink ranging, the downlink ranging + telemetry mode and the uplink distance + remote control, downlink ranging + telemetry mode. Adjust the output signal power of the star docking device and the upstream and downstream signal attenuator so that the upstream and downstream signals are the medium level and the threshold level respectively. Station measurement and control equipment for speed measurement, collecting 500 points velocity data for processing, statistical speed random error. As shown in Table 1, the satellite and the station equipment is relatively static, but in different modes, the speed results are about $-136 \mathrm{~m}$ / s system error.

Table 1. Speed performance test record table

\begin{tabular}{|c|c|c|c|c|}
\hline \multirow{2}{*}{ Signal level } & \multicolumn{2}{|c|}{ Telemetry + ranging } & \multicolumn{2}{c|}{ Telemetry + control + ranging } \\
\cline { 2 - 5 } & $\begin{array}{c}\text { Mean } \\
(\mathrm{m} / \mathrm{s})\end{array}$ & $\begin{array}{c}\text { variance } \\
(\mathrm{m} / \mathrm{s})\end{array}$ & $\begin{array}{c}\text { Mean } \\
(\mathrm{m} / \mathrm{s})\end{array}$ & $\begin{array}{c}\text { variance } \\
(\mathrm{m} / \mathrm{s})\end{array}$ \\
\hline Medium intensity & -136.152 & 0.0011 & -136.152 & 0.0010 \\
\hline Threshold & -136.154 & 0.0057 & -136.154 & 0.0060 \\
\hline
\end{tabular}

According to the main parameters and indexes of velocity measurement, the parameter setting and index of star interface and station equipment are analyzed. It is concluded that the nominal frequency difference causes the error of the velocity measurement system. In this paper, a set of upper and lower pseudo - The algorithm is carried out in detail, and the error correction method of the speed system based on nominal frequency difference measurement is proposed.

\section{The Principle of Non - Coherent Velocity Measurement}

Non-coherent Doppler Velocimetry is achieved by bidirectional pseudo-Doppler. The upstream and downstream signals adopt the measurement frame structure, the uplink measurement frame may not modulate the information, and the downlink measurement frame modulation transponder status information includes the pseudo Doppler measurement information. In order to realize the non- 
coherent velocity measurement, it is required to receive and transmit the channel clock common source and pseudo Code clock and information bit clock source, as shown in Figure 1.

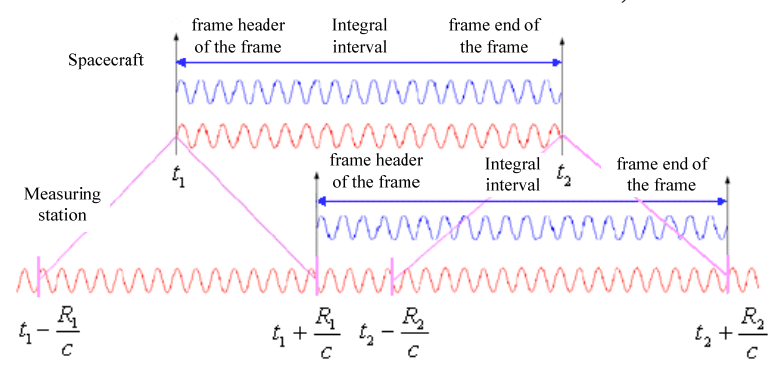

Figure 1. Non - coherent velocity diagram

The station calculates the pseudo-Doppler measurements transmitted by the satellite and the measured pseudo-Doppler measurements measured at the station to complete the measurement of the speed and frequency difference (the time between the two frames on the star) ). The sampling time of the star is the time of the frame measurement synchronization of the downlink measurement, and the sampling time of the station is the time of receiving the downlink measurement frame. It is required that the star pseudo-Doppler measurement integral data segment and the station pseudoDoppler measurement integral data segment are consistent, That is, the use of fixed time on the star cycle, the station uses a fixed cycle time measurement method.

\section{Analysis of Star Rating Frequency Difference}

\subsection{Analysis of Upstream Pseudo - Doppler Measurement}

When the number of workstations is X, the downlink measurement frame contains a set of channel Doppler information for each frame. If the previous sampling time is $t_{1}$, the current sampling time is $t_{2}$, and the on-board velocity measurement time is $t_{1} \sim t_{2}, A$ frame period for the baseline. At the time of $t_{1}$, the distance between the stations is $R_{1}$ and $t_{2}$, and the distance between the stations is $R_{2}$ and the distance between $R_{2}$ and t2. The actual radial velocity is $\left(R_{2}-R_{1}\right) /\left(t_{2}-t_{1}\right)$; the station clock error is $\sigma_{g}(t)$, the station nominal frequency is $f_{U g}$, the length of time is $t_{2}-t_{1}-\left(R_{2}-R_{1}\right) / c$, the carrier phase increment of the time period is:

$$
\int_{t_{1}-R_{1} / c}^{t_{2}-R_{2} / c} f_{U g} \times\left(1+\sigma_{g}(t)\right) d t
$$

The frequency of the station is:

$$
\frac{f_{U g}}{t_{2}-t_{1}-\left(R_{2}-R_{1}\right) / c} \times\left(\int_{t_{1}-R_{1} / c}^{t_{2}-R_{2} / c}\left(1+\sigma_{g}(t)\right) d t\right)
$$

This period of time to reach the star of the time period for the $t_{1} \sim t_{2}$, because the carrier phase increment unchanged, the length of time to $\mathrm{t}_{2} \sim \mathrm{t}_{1}$, the frequency becomes:

$$
\frac{f_{U g}}{t_{2}-t_{1}} \times\left(\int_{t_{1}-R_{1} / c}^{t_{2}-R_{2} / c}\left(1+\sigma_{g}(t)\right) d t\right)
$$

The answering machine measured the upstream signal frequency with reference to the clock frequency on the star as:

$$
f_{U g} \times\left(\int_{t_{2}-R_{1} / c}^{t_{2}-R_{2} / c}\left(1+\sigma_{g}(t)\right) d t\right) /\left(\int_{t_{1}}^{t_{2}}\left(1+\sigma_{s}(t)\right) d t\right)
$$

\subsection{Analysis of down-phase Pseudo-Doppler Measurement}

Satellite on the downlink signal time period is $t_{1} \sim t_{2}$, can be obtained satellite transponder downlink frequency:

$$
\left(\frac{f_{D s}}{t_{2}-t_{1}}\right) \times\left(\int_{t_{1}}^{t_{2}}\left(1+\sigma_{s}(t)\right) d t\right)
$$


The arrival of the station down signal phase increment, the length of time to $t_{2}-t_{1}+\left(R_{2}-R_{1}\right) / c$, the frequency is:

$$
\left(\frac{f_{D s}}{t_{2}-t_{1}+\left(R_{2}-R_{1}\right) / c}\right) \times\left(\int_{t_{1}}^{t_{2}}\left(1+\sigma_{s}(t)\right) d t\right)
$$

The measured frequency of the downstream signal is measured with reference to the clock frequency of the station as:

$$
f_{D s} \times\left(\int_{t_{1}}^{t_{2}}\left(1+\sigma_{s}(t)\right) d t\right) /\left(\int_{t_{1}+R_{1} / c}^{t_{2}+R_{2} / c}\left(1+\sigma_{g}(t)\right) d t\right)
$$

\subsection{Analysis of the Calculation Result of Velocity and the Influence of Nominal Frequency Difference}

Finishing, the composition of the speed calculation equation group:

$$
\begin{aligned}
& f_{U g} \times\left(\int_{t_{1}-R_{1} / c}^{t_{2}-R_{2} / c}\left(1+\sigma_{g}(t)\right) d t\right)=\left(f_{U s}+f_{U d}\right) \times\left(\int_{t_{1}}^{t_{2}}\left(1+\sigma_{s}(t)\right) d t\right) \\
& f_{D s} \times\left(\int_{t_{1}}^{t_{2}}\left(1+\sigma_{s}(t)\right) d t\right)=\left(f_{D g}+f_{D d}\right) \times\left(\int_{t_{1}+R_{1} / c}^{t_{2}+R_{2} / c}\left(1+\sigma_{g}(t)\right) d t\right)
\end{aligned}
$$

Eliminate the downstream measurement frame period, you can get:

$$
f_{U g} \times\left(\int_{t_{1}-R_{1} / c}^{t_{2}-R_{2} / c}\left(1+\sigma_{g}(t)\right) d t\right) /\left(f_{U s}+f_{U d}\right)=\left(f_{D g}+f_{D d}\right) \times\left(\int_{t_{1}+R_{1} / c}^{t_{2}+R_{2} / c}\left(1+\sigma_{g}(t)\right) d t\right) / f_{D s}
$$

The stability criterion of the unified carrier system is composed of cesium atomic clock, high stability crystal and phase-locked loop. The long-term stability index is better than $1 \times 10^{-\mathrm{X}}$, and the short stabilization index is better than $1 \times 10^{-\mathrm{X}}$, the station clock error is in the measurement frame Cycle error, the error is very small, the impact of the results can be ignored, therefore, on the approximate approximation, the solution was:

$$
v=\frac{R_{2}-R_{1}}{t_{2}-t_{1}}=\left[\frac{f_{U g} \times f_{D s}-\left(f_{U s}+f_{U d}\right) \times\left(f_{D g}+f_{D d}\right)}{f_{U g} \times f_{D s}+\left(f_{U s}+f_{U d}\right) \times\left(f_{D g}+f_{D d}\right)}\right] \times C
$$

According to the above calculation can be found in the speed calculation results and star clock performance has nothing to do.

\section{Error Correction of Non - Coherent Velocity Measurement System}

In this paper, the basic idea of non-coherent velocity system error correction is to make the data in engineering practice consistent with the input in the theoretical calculation method, and to do some equivalent transformation and intermediate measurement if necessary.

\subsection{Discussion on Error Correction Method}

If you can directly get the nominal frequency of the star or through the measurement to obtain accurate star nominal frequency, there are three kinds of non-coherent velocity system error correction method:

\subsubsection{Nominal Frequency Alignment}

The nominal frequency of the upper and lower frequency converters is the same as that of the satellite upstream standard, so that the simplified processing in the speed measurement formula of the existing baseband equipment is consistent with the actual situation, thus eliminating the influence of the generalized satellite frequency difference. The method requires that the base station of the station and the up-converter or down-converter have sufficient step resolution. At present, the baseband frequency of the far-board USB system is set to X Hz, the stepping accuracy of the upconverter is XXX Hz, the down- Into the accuracy of X kHz. If this method is adopted, it is necessary to transform the existing up-converter or down-converter; otherwise it is difficult to achieve high speed accuracy. 


\subsubsection{Improvement of Velocity Calculation Method}

In the baseband parameter setting, the upper and lower nominal frequency of the satellite is added, and the nominal frequency or the measured nominal frequency is set. The formula of the speed result in this paper is used to eliminate the influence of the star frequency difference. If this method is adopted, it is necessary to upgrade the parameters in the existing baseband and the software of the velocity measurement part.

\subsubsection{Upward Doppler Compensation}

If there is only the uplink nominal frequency difference, the star upstream frequency difference can be placed in the baseband uplink Doppler compensation function module to compensate for the frequency of the inverter frequency step set the accuracy of the system error, Defects in existing algorithms of baseband. The method does not need to change the existing equipment, by giving a given uplink frequency difference or the actual measurement data after the binding to the baseband Doppler dynamic analog module, and open the Doppler compensation function, you can speed system error correction The

\subsection{Standardized Frequency Difference Measurement}

If there is a certain error between the nominal frequency provided by the station and the satellite and the measured real frequency, the above three methods introduce errors. In order to more accurately achieve the non-coherent velocity or to verify the nominal frequency accuracy, the method of measuring the nominal frequency difference of the star earth under static conditions:

The frequency difference that can be measured in the actual case includes the frequency difference caused by the difference between the satellite frequency reference and the nominal frequency difference with respect to the station frequency reference.

The following proposed to normalize the satellite downstream frequency normalized to the station nominal downlink frequency, measuring satellite nominal uplink frequency for the speed system error correction. Combine $\mathrm{A}=0$ with equations (8) and (9):

$$
f_{U s}=\frac{f_{U g} \times f_{D g}}{f_{D g}+f_{D d}}-f_{U d}
$$

The method of normalization of the downlink frequency not only avoids the problem of ambiguity measurement, but also makes the proposed method of increasing the velocity correction of the uplink Doppler compensation. In the case of the nominal frequency difference The speed system error can be corrected by means of measurement, calculation and then compensation.

\section{Summary}

Based on the principle of non-coherent velocity measurement, this paper analyzes the causes of the error of the velocity measurement system caused by the nominal frequency inconsistency, and puts forward the uplink Doppler compensation correction method based on the nominal frequency difference measurement. Without changing the hardware and software The case has completed the error correction.

\section{References}

[1]. Huang Kai. XX 17 satellite satellite docking implementation of the program [R] .2016.

[2]. Wu Guangzhi .YUANWANG 5 ship TS-XXXX system XX seventeen days docking results [R] .2016. 